\title{
Targeting mTOR Complexes in Ovarian Cancer
}

Seiji Mabuchi*, Tomoyuki Sasano and Mahiru Kawano

Department of Obstetrics and Gynecology, Osaka University Graduate School of Medicine, Japan

\begin{abstract}
The mammalian (mechanistic) target of rapamycin (mTOR) is a serine/threonine kinase that plays a key role in cell growth and proliferation and is regarded as an attractive therapeutic target for cancer therapy. Preclinical investigations have suggested that mTOR complex 1 (mTORC1) and mTORC2 are frequently activated in epithelial ovarian cancer, especially in clear cell carcinoma of the ovary. In mouse models of ovarian cancer, mTORC1 inhibitors have demonstrated promising antitumor activity against ovarian cancer both in the setting of monotherapy and when used in combination with cytotoxic agents. Based on these promising preclinical findings, mTORC1 inhibitors are currently being evaluated in phase I/II trials involving ovarian cancer patients. In an effort to overcome resistance to mTORC1 inhibitors, novel mTOR kinase inhibitors (TORKinib) that inhibit both mTORC1 and mTORC2 have recently been developed. In this report, we review the scientific rationale and evidence for the potential clinical benefits provided by mTOR inhibitors in patients with epithelial ovarian cancer.
\end{abstract}

Keywords: mTORC1; mTORC2; Ovarian cancer; Rapalog; TORKinib

Abbreviations: CCC: Clear Cell Carcinoma; SAC: Serous Adenocarcinoma; mTOR: Mammalian Target of Rapamycin; mTORC: mTOR Complex; S6K-1: ribosomal S6 kinase-1; 4E-BP1: elF4E Binding Protein 1; PI3K: Phosphatidylinositol 3-kinase; Cisplatin: cisdiaminodichloroplatinum; PTEN: Phosphatase and Tensin Homolog Deleted on Chromosome 10

\section{Introduction}

Despite recent advances in surgery and chemotherapy, ovarian cancer patients are still at significant risk of recurrence, and recurrent disease can not be cured with current standard treatments. Thus, there is an urgent need to develop novel treatments based on the distinct molecular background of ovarian cancer.

The mammalian (mechanistic) target of rapamycin (mTOR) is a serine/threonine kinase that plays a key role in cell growth and proliferation $[1,2]$. In cells, mTOR acts as the catalytic subunit of two functionally distinct complexes, mTOR complex 1 (mTORC1) and mTOR complex 2 (mTORC2). Since the mTOR signaling pathway is hyperactivated in a wide range of tumor types including ovarian cancer, it is expected that mTOR inhibitors would exhibit broad therapeutic activity $[1,2]$.

Rapamycin, a natural product isolated from the bacterium Streptomyces hygroscopicus, acts as an allosteric inhibitor of mTORC1. Through the specific inhibition of mTORC1 activity, rapamycin and its derivatives (rapalogs) display multifunctional biological activity profiles; i.e., they downregulate angiogenesis and cell proliferation, growth, survival, motility, and differentiation $[1,2]$.

mTOR kinase inhibitors (TORKinibs) are newly developed ATPcompetitive inhibitors of mTOR that can inhibit both mTORC1 and mTORC2. TORKinibs have been shown to inhibit mTOR signaling more strongly than rapamycin by suppressing both mTORC1 and mTORC2, attenuating protein synthesis, and inducing cell cycle arrest and/or apoptosis in cancer cells [3].

On the basis of promising preclinical data, these mTOR inhibitors are currently being evaluated in phase I/II trials in patients with ovarian cancer. This article highlights the scientific rationale for mTOR- targeting therapies against ovarian cancer treatment and summarizes the available preclinical and clinical findings.

\section{mTOR: Structure and Function}

mTORC1 is composed of six proteins: $\mathrm{mTOR}$, raptor (regulatoryassociated protein of mTOR), mLST8/G $\beta \mathrm{L}$ (mammalian LST8/Gprotein $\beta$ - subunit like protein), PRAS40 (proline-rich AKT substrate of $40 \mathrm{kDa}$ ), and DEPTOR (DEP domain-containing mTOR-interacting protein). In response to extracellular signals, $\mathrm{PI} 3 \mathrm{~K}$ (phosphatidylinositol3-kinase) phosphorylates PIP2 (phosphatidylinositol 4,5-bisphosphate) to generate PIP3 (phosphatidylinositol $(3,4,5)$-triphosphate), leading to the recruitment of AKT to the plasma membrane, where it is phosphorylated and activated (Figure 1). Activated AKT can directly activate mTORC1 by phosphorylating mTOR at Ser 2448, and can indirectly activate mTORC1 by phosphorylating TSC2 (tuberous sclerosis complex 2, also called tuberin). The phosphorylation of TSC2 by AKT leads to the inhibition of the function of the TSC1/TSC2 complex. When TSC1/TSC2 is active, TSC2 stimulates the conversion of Rheb (Ras homolog enriched in brain)-GTP to Rheb-GDP, which inactivates mTORC1. When TSC2 is phosphorylated and inactivated by AKT, Rheb-GTP stimulates the activity of mTORC1. Therefore, genetic alterations involving this signaling pathway lead to increased activation of mTORC1 [1,2].

Once activated, mTORC1 phosphorylates the translationregulating factors S6K-1 (ribosomal S6 kinase-1) and 4EBP-1 (eukaryote translation initiation factor $4 \mathrm{E}$ binding protein-1). The activation of S6K-1 leads to the translation of mRNA encoding ribosomal proteins, elongation factors, and other proteins required for transition from the G1 phase to the $S$ phase of the cell cycle $[1,2]$. The phosphorylation of

*Corresponding author: Seiji Mabuchi, M.D., Ph.D., Department of Obstetrics and Gynecology, Osaka University Graduate School of Medicine, 2-2 Yamadaoka, Suita, Osaka 565-0871, Japan, Tel: +81-6-6879-3354; Fax: +81-6-6879-3359; E-mail: smabuchi@gyne.med.osaka-u.ac.jp

Received April 18, 2014; Accepted June 19, 2014; Published June 23, 2014

Citation: Mabuchi S, Sasano T, Kawano M (2014) Targeting mTOR Complexes in Ovarian Cancer. J Cancer Sci Ther 6: 211-216. doi:10.4172/1948-5956.1000274

Copyright: (c) 2014 Mabuchi S, et al. This is an open-access article distributed under the terms of the Creative Commons Attribution License, which permits unrestricted use, distribution, and reproduction in any medium, provided the original author and source are credited. 


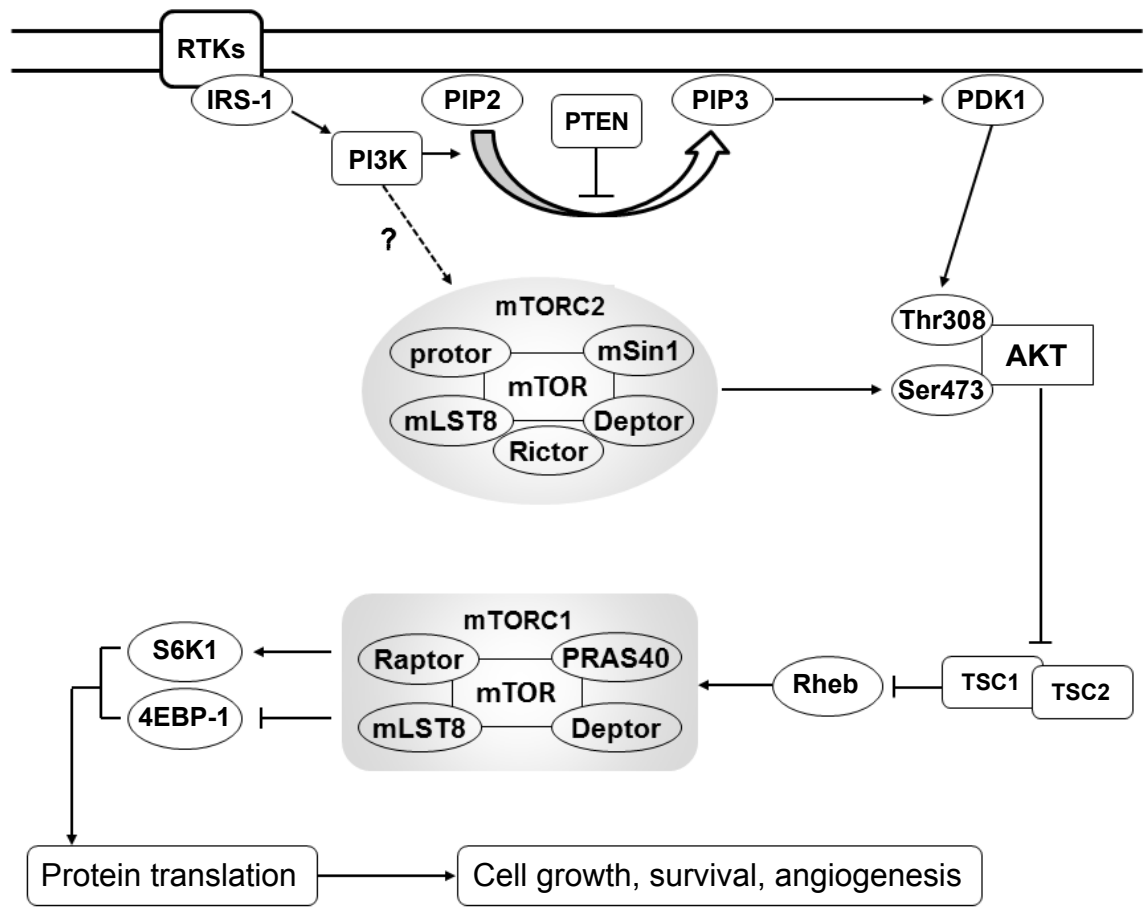

Figure 1: Schematic representation of the mTOR signaling pathway.

4EBP-1 also enhances the translation of mRNA encoding cyclin D1, c-Myc, and hypoxia-inducible factor-1 $\alpha$ (HIF-1 $\alpha$ ), leading to cell cycle progression or angiogenesis [1,2].

The other mTOR complex, mTORC2, consists of six proteins: mTOR, rictor (rapamycin-insensitive companion of mTOR), mSIN1 (mammalian SAPK interacting protein), protor (protein observed with rictor), $\mathrm{mLST} 8 / \mathrm{G} \beta \mathrm{L}$, and DEPTOR [1]. The precise mechanism by which mTORC2 is activated remains unclear. However, a recent report suggested that PI3K is required to activate mTORC2 [4]. Activated mTORC2, in which mTOR is phosphorylated at Ser 2481 [5], in turn phosphorylates AGC kinases, such as AKT, serum and glucocorticoidregulated kinases (SGK), and protein kinase C- $\alpha$ (PKC- $\alpha$ ), and controls cell growth by regulating lipogenesis, glucose metabolism, the actin cytoskeleton, and apoptosis [6].

\section{mTORC1 Inhibitors}

Rapamycin (sirolimus), a potent inhibitor of mTORC1, was isolated in 1975 from the bacterium Streptomyces hygroscopicus [7]. Rapamycin inhibits mTORC1 by first binding to the intracellular protein FK506 binding protein 12 (FKBP12). The resultant rapamycinFKBP12 complex then binds to the FKBP12-rapamycin-binding domain (FRB) of mTORC1 and inhibits the serine/threonine kinase activity of mTORC1 via an allosteric mechanism. In contrast to mTORC1, the rapamycin-FKBP12 complex cannot interact with the FRB domain of mTORC2, and thus, mTORC2 is generally resistant to rapamycin treatment [1].

As rapamycin exhibits very poor solubility in water, which limits its clinical use, several soluble ester analogs of rapamycin (rapalogs) have been developed [2]. Currently, these analogs include temsirolimus, everolimus, and ridaforolimus. Temsirolimus and everolimus are formulated for i.v. administration and oral administration, respectively.
Ridaforolimus was initially developed as an i.v. formulation, but an oral formulation was subsequently developed [8].

\section{mTORC1 in Ovarian Cancer: Preclinical Findings}

\section{mTORC1 activation in ovarian cancer}

It has previously been reported that somatic mutations in PTEN, PIK3CA, PIK3R1, and AKT1 are observed in 9.8\% [9], 12\% [10], 3.8\% [11], and $2 \%$ [12] of ovarian cancers, respectively. Moreover, recent studies of The Cancer Genome Atlas (TCGA) have identified mTOR mutations in $1.9 \%$ of ovarian cancers [13]. Any one of these genetic changes can lead to increased activation of mTORC1 signaling in ovarian cancer.

Altomare et al. were the first to demonstrate that mTORC1 activation is common in human ovarian cancer. In the article, it was reported that mTORC1 was activated in 55\% (17 of 31) of epithelial ovarian cancers [14]. Subsequently, Mabuchi et al. examined 98 primary ovarian cancers [ 52 clear cell carcinomas (CCC) and 46 serous adenocarcinomas (SAC)] using tissue microarrays and reported that mTORC1 is more frequently activated in CCC than SAC ( $86.6 \%$ vs $50 \%)$ [15]]. The frequent mTORC1 activation observed in CCC can be explained, at least in part, by the fact that PIK3CA-activating mutations occur more frequently in CCC than in other histological subtypes of epithelial ovarian cancer [16].

\section{Therapeutic potential of mTORC1 inhibitors in ovarian cancer}

In the setting of monotherapy, treatment with mTORC1 inhibitors effectively attenuated cell proliferation, tumor growth, and angiogenesis in ovarian cancer both in vitro and in vivo $[17,18]$. mTORC1 inhibitors have also been successfully combined with chemotherapeutic agents including cisplatin [18], paclitaxel [19], 
carboplatin [20], and trabectedin [21]. Furthermore, mTORC1 inhibitors have shown promising activity when used in combination with biological agents including bevacizumab [22], mitogen-activated protein kinase kinase (MEK) inhibitors [23], or epidermal growth factor receptor (EGFR) inhibitors [24]. These findings indicate that combining mTORC1 inhibition with the use of cytotoxic or biological agents could be a potentially effective treatment for ovarian cancer. Moreover, given their significant anti-proliferative and anti-angiogenic effects mTORC1 inhibitors might be useful as a maintenance therapy for preventing or delaying the development of recurrent disease after front-line chemotherapy.

Importantly, the growth-inhibiting effect of everolimus was marked in cells that exhibited high AKT/mTORC1 activity, whereas it was minimal in cells displaying low AKT/mTORC1 activity $[17,18]$. These results indicate that ovarian cancer cells with elevated AKT/ mTORC1 activity are more susceptible to mTORC1 inhibitors than cells displaying low AKT/mTORC1 activity. Thus, patients with CCC might be most responsive to mTORC1-targeting therapy.

\section{Clinical trials of mTORC1 inhibitors}

On the basis of promising preclinical findings, temsirolimus, everolimus, and ridaforolimus are currently being tested in phase I/II clinical trials involving ovarian cancer patients, either as single agents or in combination with cytotoxic or biological agents (Table 1). Of these, the only study that has published results is the GOG phase II trial of temsirolimus (GOG 170-I). In this trial, temsirolimus monotherapy was evaluated in patients with persistent or recurrent epithelial ovarian or primary peritoneal malignancies [25]. Of the 60 enrolled patients, 54 were eligible for evaluation. Of these, 9.3\% experienced a partial response, and $24.1 \%$ achieved progression-free survival (PFS) periods of $\geq 6$ months, which was just below the threshold used to determine inclusion in phase III studies [25]. In this study, the patients with ovarian tumors that exhibited mTORC1 activity achieved a higher response rate than those whose tumors did not display mTORC1 activity although the difference was not statistically significant (PFS $\geq 6$ months, $30.3 \%$ vs $11.8 \%$; response rate: $11.8 \%$ vs $5.9 \%$ ).

The clinical activity of mTORC1 is currently being evaluated in patients with ovarian CCC, a chemoresistant histological subtype characterized by frequent hyperactivation of mTORC1. The Gynecologic Oncology Group (GOG) is currently conducting a phase II trial (protocol GOG0268) of combination treatment involving temsirolimus combined with carboplatin and paclitaxel followed by temsirolimus consolidation therapy as a first-line therapy in patients with stage III-IV CCC of the ovary [26]. In addition, the Japanese Gynecologic Oncology Group (JGOG) has just initiated a phase II trial examining the utility of everolimus monotherapy as a treatment for recurrent CCC of the ovary (protocol JGOG3012).

\begin{tabular}{|c|c|c|c|c|}
\hline Studies $^{1}$ & Target & Condition & Interventions & Phase \\
\hline \multicolumn{5}{|l|}{ Temsirolimus } \\
\hline NCT00926107 & Ovarian cancer & Recurrent & Temsirolimus & Phase II \\
\hline NCT00429793 & $\begin{array}{c}\text { Ovarian cancer } \\
\text { Fallopian tube cancer } \\
\text { Primary peritoneal cancer }\end{array}$ & Recurrent & Temsirolimus & Phase II \\
\hline $\begin{array}{l}\text { NCT01196429 } \\
\text { GOG0268 }\end{array}$ & $\begin{array}{l}\text { Clear cell ovarian cancer } \\
\text { (Stage III-IV) }\end{array}$ & First-line therapy & $\begin{array}{l}\text { Temsirolimus plus carboplatin/paclitaxel flowed by } \\
\text { temsirolimus consolidation }\end{array}$ & Phase II \\
\hline NCT01460979 & $\begin{array}{l}\text { Ovarian cancer } \\
\text { Endometrial cancer }\end{array}$ & Recurrent & Temsirolimus & Phase II \\
\hline NCT01010126 & Solid tumors & Advanced or recurrent & Temsirolimus plus bevacizumab & Phase II \\
\hline NCT00982631 & $\begin{array}{l}\text { Ovarian cancer } \\
\text { Breast cancer } \\
\text { Endometrial cancer }\end{array}$ & Advanced or recurrent & Temsirolimus plus PLD & Phase I \\
\hline NCT00408655 & Solid tumors & Advanced & Temsirolimus plus carboplatin/paclitaxel & Phase I \\
\hline NCT00523432 & Gynecologic malignancies & Recurrent & Temsirolimus plus topotecan & Phase I \\
\hline NCT00703170 & Solid tumors & Recurrent & Temsirolimus plus PLD & Phase I \\
\hline NCT01155258 & Solid tumors & Advanced & Temsirolimus plus vinorelbine ditartrate & Phase I \\
\hline NCT00703625 & Solid tumors & Recurrent & Temsirolimus plus docetaxel & Phase I \\
\hline NCT01065662 & Gynecologic malignancies & Recurrent & Temsirolimus plus cediranib & Phase I \\
\hline \multicolumn{5}{|l|}{ Everolimus } \\
\hline NCT01149434 & Solid tumors & Advanced & $\begin{array}{c}\mathrm{Jl}-101 \text { vs } \\
\mathrm{Jl}-101 \text { plus everolimus }\end{array}$ & Phase I/II \\
\hline NCT01031381 & $\begin{array}{c}\text { Ovarian cancer } \\
\text { Fallopian tube cancer } \\
\text { Primary peritoneal cancer }\end{array}$ & Recurrent & Everolimus plus bevacizumab & Phase II \\
\hline NCT00886691 & $\begin{array}{c}\text { Ovarian cancer } \\
\text { Fallopian Tube Cancer } \\
\text { Primary Peritoneal Cancer }\end{array}$ & Recurrent & $\begin{array}{c}\text { Bevacizumab vs } \\
\text { bevacizumab plus everolimus }\end{array}$ & Phase II \\
\hline JGOG3021 2 & Clear cell ovarian cancer & Recurrent & Everolimus & Phase II \\
\hline \multicolumn{5}{|l|}{ Ridaforolimus } \\
\hline NCT01256268 & $\begin{array}{l}\text { Ovarian cancer } \\
\text { Endometrial cancer }\end{array}$ & Recurrent or advanced & Ridaforolimus plus carboplatin/paclitaxel & Phase I \\
\hline NCT01295632 & Solid tumors & Advanced & Ridaforolimus & Phase I \\
\hline
\end{tabular}

${ }^{1}$ ClinicalTrials.gov identifier.

${ }^{2}$ This study will be initiated from July 2014

PLD; pegylated liposomal doxorubicin hydrochloride, GOG; Gynecologic Oncology Group, JGOG; Japanese Gynecologic Oncology Group.

Table 1: Summary of mTORC1 inhibitors in clinical trials. 


\section{Common Toxicities Associated with mTORC1 Inhibitors}

mTORC1 inhibitors are generally well tolerated, with their most common side effects including stomatitis, rash, fatigue, hyperglycemia, hyperlipidemia, hypercholesterolemia, and myelosuppression. Most of these side effects are mild and can be resolved by dose interruption or reduction. However, whilst symptomatic non-infectious pneumonitis is a relatively uncommon class effect of mTOR inhibitors it can be life-threatening. In a phase III clinical trial of temsirolimus involving renal cell carcinoma patients, asthenia, rash, and anemia were found to be the most common side effects, with incidences of 51, 47, and $45 \%$, respectively [27]. In addition, a significant percentage of the patients presented with metabolic disturbances such as hyperlipidemia (27\%), hyperglycemia (26\%), or hypercholesterolemia (24\%). Respiratory symptoms such as coughing and dyspnea were reported in approximately a quarter of the patients ( 26 and $28 \%$, respectively) and stomatitis was observed in about $20 \%$.

\section{Mechanism Responsible for Resistance to mTORC1 Inhibitors}

The major limitation affecting mTORC1-targeting therapy is that drug resistance can develop, which often results in disease relapse. The mechanisms responsible for acquired resistance to mTORC1 inhibitors might involve the loss of the negative feedback loops that are normally induced when mTORC1 is active (Figure 2). Regarding these negative feedback loops, the first involves mTORC2-independent IRS1-dependent AKT activation in response to mTORC1 inhibition [28], the second involves mTORC2-mediated AKT activation in response to treatment with $\mathrm{mTORC} 1$ inhibitors [3], and the third involves mTORC1 inhibition-mediated mitogen-activated protein kinase (MAPK) activation [29]. Thus, novel inhibitors that target both mTORC1 and mTORC2 or the use of a combination of mTORC1targeting agents and MAPK inhibitors might have the potential to overcome resistance to mTORC1 inhibitors. However, it remains unknown whether these mechanisms are cell type-specific and whether the abovementioned preclinical findings are of clinical relevance to ovarian cancer.

\section{Molecular Biomarkers that could be used during mTORC1-Tageted Therapy}

There is an urgent need for efficient biomarkers to make it possible to precisely predict which patients will benefit from mTORC1-targeting therapies, and hence, to ensure that patients who will not benefit from such treatments can avoid unnecessary toxicities. Moreover, the identification of surrogate markers that are useful for monitoring the activity of mTORC1 inhibitors is also necessary. Previous preclinical and clinical investigations have suggested that cells carrying PIK3CA mutations [30], hyper activation of AKT/mTORC1 signaling, and the overexpression of cyclin D1 are particularly sensitive to mTORC1 inhibitors [31]. Moreover, the overexpression of Bcl-2 [32] and KRAS mutations might also serve as predictors of resistance to mTORC1 inhibitors [33]. However, the clinical utility of these candidate biomarkers needs to be validated in patients with ovarian cancer.

\section{mTORC2 in Ovarian Cancer}

A recent report suggested that rictor, an essential component of mTORC2, is frequently expressed in ovarian cancer [3]. Moreover, mTORC2 was found to be more frequently activated in CCC than in SAC $(71.2 \%$ versus $45.7 \%$, respectively). Thus, strategies aimed at inhibiting the activity of mTORC2 might play important roles as treatments for ovarian cancer, especially ovarian CCC [3].

Recently, mTOR kinase inhibitors (TORKinibs), which can inhibit both mTORC1 and mTORC2 complexes, have been developed [34]. TORKinibs have been shown to inhibit mTOR signaling by suppressing both mTORC1 and mTORC2, attenuate protein synthesis, and induce cell cycle arrest and/or apoptosis more strongly than mTORC1 inhibitors in cancer cells $[3,35]$. However, as TORKinibs are ATP-competitive kinase inhibitors issues with their selectivity and off-
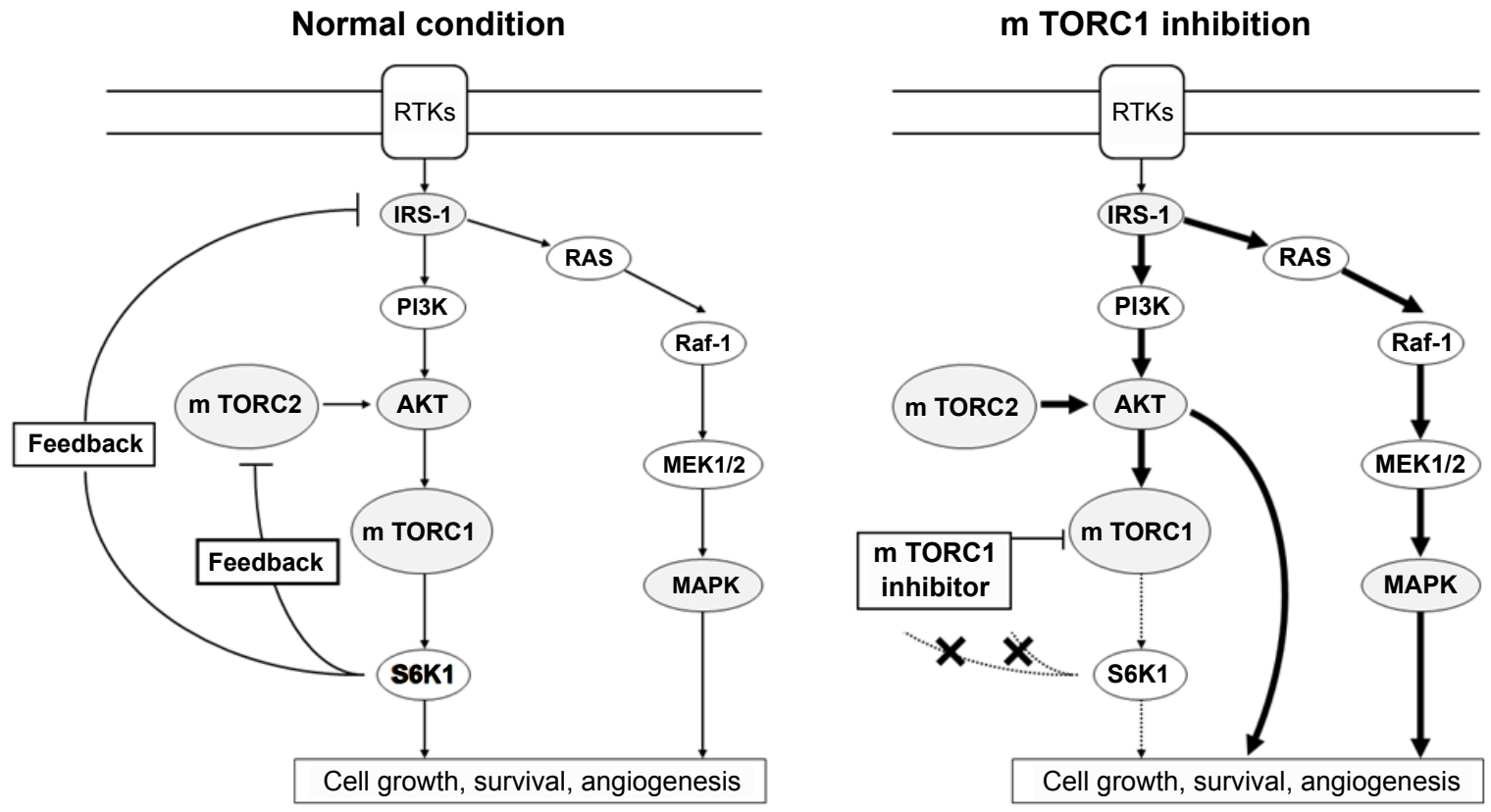

Figure 2: The mechanisms responsible for resistance to mTORC1 inhibitors 
target effects are inevitable, and thus, they might be associated with more severe toxicities than mTORC1 inhibitors. The clinical activity and toxicities of TORKinibs are currently being examined in phase I/II clinical trials involving patients with solid malignancies [36].

\section{Conclusions and Future Directions}

mTOR complexes are frequently activated in epithelial ovarian cancer, especially in CCC of the ovary. On the basis of promising preclinical findings, mTORC1 inhibitors are currently being evaluated as treatments for ovarian cancer, either alone or in combination with chemotherapeutic agents. Considering the mechanism responsible for resistance to $\mathrm{mTORC} 1$ inhibitors, greater success might also be possible with novel molecules that target both mTORC1 and mTORC2. Given the potential toxicity of mTOR-targeting agents, it is more important than ever to identify biomarkers that can be used to predict which patients will benefit from such agents. Overcoming these challenges will aid the development of optimal personalized mTOR-targeting therapies for ovarian cancer.

\section{Acknowledgements}

We thank Yuko Nishimura for providing secretarial assistance.

This study was supported by a grant-in-aid for General Scientific Research from the Ministry of Education, Culture, Sports, Science and Technology of Japan (no. 26462523).

The authors declare that they have no conflicts of interest.

\section{References}

1. Guertin DA, Sabatini DM (2007) Defining the role of mTOR in cancer. Cancer Cell 12: 9-22.

2. Sabatini DM (2006) mTOR and cancer: insights into a complex relationship. Nat Rev Cancer 6: 729-734

3. Hisamatsu T, Mabuchi S, Matsumoto Y, Kawano M, Sasano T, et al. (2013) Potential role of mTORC2 as a therapeutic target in clear cell carcinoma of the ovary. Mol Cancer Ther 12: 1367-1377.

4. Zinzalla V, Stracka D, Oppliger W, Hall MN (2011) Activation of mTORC2 by association with the ribosome. Cell 144: 757-768.

5. Copp J, Manning G, Hunter T (2009) TORC-specific phosphorylation of mammalian target of rapamycin (mTOR): phospho-Ser2481 is a marker for intact mTOR signaling complex 2. Cancer Res 69: 1821-1827.

6. Laplante M, Sabatini DM (2012) mTOR signaling in growth control and disease. Cell 149: 274-293.

7. Sehgal SN, Baker H, Vézina C (1975) Rapamycin (AY-22,989), a new antifungal antibiotic. II. Fermentation, isolation and characterization. J Antibiot (Tokyo) 28: 727-732.

8. Mahalingam D, Sankhala K, Mita A, Giles FJ, Mita MM (2009) Targeting the mTOR pathway using deforolimus in cancer therapy. Future Oncol 5: 291-303.

9. Obata K, Morland SJ, Watson RH, Hitchcock A, Chenevix-Trench G, et al. (1998) Frequent PTEN/MMAC mutations in endometrioid but not serous or mucinous epithelial ovarian tumors. Cancer Res 58: 2095-2097.

10. Levine DA, Bogomolniy F, Yee CJ, Lash A, Barakat RR, et al. (2005) Frequent mutation of the PIK3CA gene in ovarian and breast cancers. Clin Cancer Res 11: $2875-2878$.

11. Philp AJ, Campbell IG, Leet C, Vincan E, Rockman SP, et al. (2001) The phosphatidylinositol 3'-kinase p85alpha gene is an oncogene in human ovarian and colon tumors. Cancer Res 61: 7426-7429.

12. Carpten JD, Faber AL, Horn C, Donoho GP, Briggs SL, et al. (2007) A transforming mutation in the pleckstrin homology domain of AKT1 in cancer. Nature 448: 439-444

13. cBioPortal for Cancer Genomics.

14. Altomare DA, Wang HQ, Skele KL, De Rienzo A, Klein-Szanto AJ, et al. (2004)
AKT and mTOR phosphorylation is frequently detected in ovarian cancer and can be targeted to disrupt ovarian tumor cell growth. Oncogene 23: 5853-5857.

15. Mabuchi S, Kawase C, Altomare DA, Morishige K, Sawada K, et al. (2009) mTOR is a promising therapeutic target both in cisplatin-sensitive and cisplatinresistant clear cell carcinoma of the ovary. Clin Cancer Res 15: 5404-5413.

16. Kuo KT, Mao TL, Jones S, Veras E, Ayhan A, et al. (2009) Frequent activating mutations of PIK3CA in ovarian clear cell carcinoma. Am J Pathol 174: 15971601.

17. Mabuchi S, Altomare DA Connolly DC, Klein-Szanto A, Litwin S, et al. (2007) RAD001 (Everolimus) delays tumor onset and progression in a transgenic mouse model of ovarian cancer. Cancer Res 67: 2408-2413.

18. Mabuchi S, Altomare DA, Cheung M, Zhang L, Poulikakos PI, et al. (2007) RAD001 inhibits human ovarian cancer cell proliferation, enhances cisplatininduced apoptosis, and prolongs survival in an ovarian cancer model. Clin Cancer Res 13: 4261-4270.

19. Jiang H, Feng $Y$ (2006) Hypoxia-inducible factor 1alpha (HIF-1alpha) correlated with tumor growth and apoptosis in ovarian cancer. Int J Gynecol Cancer 16 Suppl 1: 405-412.

20. Schlosshauer PW, Li W, Lin KT, Chan JL, Wang LH (2009) Rapamycin by itself and additively in combination with carboplatin inhibits the growth of ovarian cancer cells. Gynecol Oncol 114: 516-522.

21. Mabuchi S, Hisamatsu T, Kawase C, Hayashi M, Sawada K, et al. (2011) The activity of trabectedin as a single agent or in combination with everolimus for clear cell carcinoma of the ovary. Clin Cancer Res 17: 4462-4473.

22. Huynh H, Teo CC, Soo KC (2007) Bevacizumab and rapamycin inhibit tumor growth in peritoneal model of human ovarian cancer. Mol Cancer Ther 6: 29592966.

23. Kinross KM, Brown DV, Kleinschmidt M, Jackson S, Christensen J, et al. (2011) In vivo activity of combined PI3K/mTOR and MEK inhibition in a Kras(G12D);Pten deletion mouse model of ovarian cancer. Mol Cancer Ther 10: $1440-1449$.

24. Glaysher S, Bolton LM, Johnson P, Atkey N, Dyson M, et al. (2013) Targeting EGFR and PI3K pathways in ovarian cancer. Br J Cancer 109: 1786-1794.

25. Behbakht K, Sill MW, Darcy KM, Rubin SC, Mannel RS, et al. (2011) Phase II trial of the mTOR inhibitor, temsirolimus and evaluation of circulating tumor cells and tumor biomarkers in persistent and recurrent epithelial ovarian and primary peritoneal malignancies: a Gynecologic Oncology Group study Gynecol Oncol 123: 19-26.

26. National Cancer Institute (2014) Temsirolimus, Carboplatin, and Paclitaxe as First-Line Therapy in Treating Patients With Newly Diagnosed Stage III-IV Clear Cell Ovarian Cancer.ClinicalTrials.gov. NCT01196429.

27. Hudes G, Carducci M, Tomczak P, Dutcher J, Figlin R, et al. (2007) Temsirolimus, interferon alfa, or both for advanced renal-cell carcinoma. N Engl J Med 356: 2271-2281.

28. Takano A, Usui I, Haruta T, Kawahara J, Uno T, et al. (2001) Mammalian target of rapamycin pathway regulates insulin signaling via subcellular redistribution of insulin receptor substrate 1 and integrates nutritional signals and metabolic signals of insulin. Mol Cell Biol 21: 5050-5062.

29. Carracedo A, Ma L, Teruya-Feldstein J, Rojo F, Salmena L, et al. (2008) Inhibition of mTORC1 leads to MAPK pathway activation through a PI3Kdependent feedback loop in human cancer. J Clin Invest 118: 3065-3074.

30. Janku F, Wheler JJ, Westin SN, Moulder SL, Naing A, et al. (2012) PI3K AKT/mTOR inhibitors in patients with breast and gynecologic malignancies harboring PIK3CA mutations. J Clin Oncol 30: 777-782.

31. Delbaldo C, Albert S, Dreyer C, Sablin MP, Serova M, et al. (2011) Predictive biomarkers for the activity of mammalian target of rapamycin (mTOR) inhibitors. Target Oncol 6: 119-124.

32. Aguirre D, Boya P, Bellet D, Faivre S, Troalen F, et al. (2004) Bcl-2 and CCND1/CDK4 expression levels predict the cellular effects of mTOR inhibitors in human ovarian carcinoma. Apoptosis 9: 797-805.

33. Janku F, Wheler JJ, Naing A, Falchook GS, Hong DS, et al. (2013) PIK3CA mutation H1047R is associated with response to PI3K/AKT/mTOR signaling pathway inhibitors in early-phase clinical trials. Cancer Res 73: 276-284.

34. Sparks CA, Guertin DA (2010) Targeting mTOR: prospects for mTOR complex 2 inhibitors in cancer therapy. Oncogene 29: 3733-3744. 
Citation: Mabuchi S, Sasano T, Kawano M (2014) Targeting mTOR Complexes in Ovarian Cancer. J Cancer Sci Ther 6: 211-216. doi:10.4172/19485956.1000274

35. Chresta CM, Davies BR, Hickson I, Harding T, Cosulich S, et al. (2010) AZD8055 is a potent, selective, and orally bioavailable ATP-competitive mammalian target of rapamycin kinase inhibitor with in vitro and in vivo antitumor activity.

Cancer Res 70: 288-298.
36. EMD Serono (2014) Trial of Pimasertib with SAR245409 or Placebo in Ovarian Cancer. ClinicalTrials.gov. NCT01936363.

This article was originally published in a special issue, Novel Targeted

Therapies for Patients with Ovarian Cancer handled by Editor(s). Dr. Samir A Farghaly, Cornell University, USA 